\title{
RADIOCARBON CALIBRATION BY THE DATE DISTRIBUTION METHOD
}

\author{
Paul Muzikar \\ Department of Physics, Purdue University, West Lafayette IN 47907, USA
}

\begin{abstract}
A method is presented for calibrating radiocarbon ages based on statistical analysis of a large number of randomly distributed dates. One interesting feature of this method is that it is internal; that is, it allows one to extend a known calibration curve further back in time by using only ${ }^{14} \mathrm{C}$ dates, with no reference to any other dating technique. A serious difficulty in implementing this method lies in assembling a sample of dates with the correct statistical properties.
\end{abstract}

\section{INTRODUCTION}

Great success has been achieved over the past few decades in calibrating the radiocarbon time scale. Tree rings have provided a very accurate calibration going back about $12,000 \mathrm{yr}$, while the U-Th dating of corals has been used to extend the calibration back to around 30,000 yr. For an up-to-date discussion of this progress, the reader is referred to Stuiver et al. (1998), along with accompanying articles in the same issue of Radiocarbon. The calibration effort has an important influence on many fields of research; thus, it is fitting to bring to bear on this effort as many independent lines of thought as possible.

My purpose in this paper is to discuss a somewhat speculative method for calibrating the ${ }^{14} \mathrm{C}$ time scale, referred to here as the Date Distribution (DD) Method. This method, as the discussion will make clear, offers only a limited resolution in time. However, it has the interesting feature of being an internal calibration method. That is, once a calibration curve is established, the DD method can extend it back in time by using only ${ }^{14} \mathrm{C}$ dates, with no necessity of using another dating method.

The word "speculative" was used in the preceding paragraph for a particular reason. A serious difficulty would have to be overcome in applying the DD method. As explained in the next section, a set of ${ }^{14} \mathrm{C}$ dates satisfying certain criteria must be assembled in order for the DD method to work. At present, it is not clear exactly how this should be done, although it is by no means demonstrably impossible. To some extent, then, this paper constitutes a "thought experiment".

In the next section I explain the DD Method, and try to highlight the various difficulties that could arise in implementing it. In the following section I present a numerical experiment illustrating how the method works. This experiment also allows us to discuss the level of precision that could be expected in any real effort to implement the method.

\section{METHOD}

To examine the DD method in some detail, we must first establish our notation. We will plot dates in the $\mathrm{X}-\mathrm{Y}$ plane, with $x$ denoting the true date (in cal BP) and $y$ denoting the conventional ${ }^{14} \mathrm{C}$ age (in BP). A calibration curve relates these two dates, and is given by

$$
y=\phi(x)
$$

The goal of calibration research is to determine the function $\phi(x)$.

Now, imagine that on a certain interval $a \leq x \leq b$, we have $\mathrm{M}$ dates distributed randomly on the $\mathrm{X}$ axis; that is, each date is equally likely to have any value between $a$ and $b$. Thus, the probability density for a given true date is given by: 


$$
p_{1}(x)=\frac{1}{b-a}
$$

for $x$ between $a$ and $b$, and is zero otherwise. For each of these dates, we actually measure $y$, the conventional age. The values of $y$ will lie between $y_{a}=\phi(a)$ and $y_{b}=\phi(b)$. We thus investigate the probability density for $y$, which we will call $p_{2}(y)$. Since for any corresponding intervals $d x$ and $d y$ we must have

$$
p_{1}(x) d x=p_{2}(y) d y,
$$

we can then say that

$$
\frac{d y}{d x}=\phi^{\prime}(x)=\frac{p_{1}}{p_{2}} .
$$

Or, with our particular form for $p_{1}(x)$,

$$
\phi^{\prime}(x)=\frac{1}{(b-a) p_{2}(y)} .
$$

The idea of the DD method is based on Equation (5). Here is how it would work. From the distribution of the actual, measured uncalibrated dates lying between $y_{a}$ and $y_{b}$, one would construct an approximation to the function $p_{2}(y)$. However, now comes a key point. To use Equation (5) to determine the slope of the calibration curve, we need to know the value of $(b-a)$; from the data we only know $y_{a}$ and $y_{b}$. To overcome this problem, we let the recent portion of our measured conventional ages overlap with a known calibration curve. Thus, we determine $(b-a)$ by fitting Equation (5) to a known portion of the calibration curve.

Note that Equation (5) only gives us the slope of the calibration curve. So, to use it to extend the calibration back in time, we need at least one point from an already existing calibration curve. Thus, in applying the DD method, we need to work from an already existing calibration curve for 2 reasons:

- because the value of $(b-a)$ is not generated by the data

- because the method only gives us the first derivative of the calibration curve.

To summarize, here are the basic steps involved in implementing the DD method:

1. Collect a large number of ${ }^{14} \mathrm{C}$ ages $y$ on an interval $y_{a}$ to $y_{b}$. These events should have a flat probability distribution for their true ages.

2. Use this data to construct an approximation to the distribution $p_{2}(y)$.

3. Fit Equation (5) to a known portion of the calibration curve to determine the value of $(b-a)$.

4. Use Equation (5) to find the slope of the calibration curve, and to extend the curve back into an unknown region of time.

Up to this point in this section we have glossed over the key difficulty (and perhaps the most interesting philosophical question) concerning the DD method. For the method to work, we must use a large sample of events whose true dates are distributed with even probability over some particular time interval. Clearly, much archaeological or geological insight is required in assembling such a sample. For example, if a sample had many dates clustered around a particular value, simply because that value was a heavily dated, interesting archaeological episode, it would be an unsuitable sample. 
Perhaps an appropriate group of dates would be a set of ${ }^{14} \mathrm{C}$ dates for various geological phenomena from all over the world. In any event, the compilation of such a sample is a question worthy of comment from knowledgeable researchers.

One other small point can be made. We have presented our discussion assuming that the function $\phi(x)$ has a positive slope for all values of $\mathrm{x}$. This is not necessarily true for realistic calibration curves. The DD method, however, will construct a quite coarse-grained version of the function, where many short-lived features are washed out. This coarse-grained function is much less likely to have a negative slope at any points.

\section{NUMERICAL EXPERIMENT}

To show how the DD method would be used in practice, let us now work through a numerical experiment. For the sake of illustration, we use a hypothetical calibration curve, a polynomial generated to have many of the features of the actual calibration curve presented by Stuiver et al. (1998). We consider the time interval, in real years, of 10,000-18,000 cal BP. For our hypothetical calibration curve, this corresponds to an interval in uncalibrated years of 9000-15,000 BP. The functional form of our curve is given by the following polynomial:

$$
\begin{aligned}
\phi(x)=.1312307 \times 10^{-6} x^{7}- & .3256363 \times 10^{-5} x^{6}-.0001273 x^{5}+.00581797 x^{4}-.0773117 x^{3} \\
+ & .303132 x^{2}+1.92271 x-6.7332 .
\end{aligned}
$$

Here, $x$ and $y$ are measured in units of ka. Figure 1 shows the calibration curve, while Figure 2 shows a plot of its first derivative. We have selected a function that has reasonable deviations from linearity, in that its slope varies from its average value by about $20 \%$. Larger deviations from linearity would be easier to see with our statistical method, while smaller deviations would be more difficult to see.

We conduct our experiment in several steps. We first use a random number generator to produce 2400 dates randomly distributed between $10,000 \mathrm{cal}$ BP and 18,000 cal BP. We then use our hypothetical calibration curve to turn these into 2400 uncalibrated dates lying between $9000 \mathrm{BP}$ and $15,000 \mathrm{BP}$. Because the calibration curve is not linear, these uncalibrated dates are not evenly distributed between the two limits. They will tend to be bunched into regions where the calibration curve has a lower slope. This, of course, is the basis of the DD method.

The idea of the experiment is to take those 2400 uncalibrated dates as our data, and then try to reconstruct the calibration curve from them. There are many particular ways to use Equation (5) to do this reconstruction. Here, we will use a simple, straightforward method. We divide up the uncalibrated period into 6 equal, 1000-yr intervals and then we count how many of the events are in each interval; Table 1 shows these numbers. From these numbers we obtain our experimental determination of $p_{i}$, the probability of an event being in 1 of the 6 intervals. For each of the 6 intervals, we estimate the average slope $s_{i}$ for that interval as

$$
s_{i}=\frac{\mathrm{A}}{p_{i}}
$$

where $\mathrm{A}$ is a constant. We thus are approximating the calibration curve as a series of line segments with slopes $s_{i}$. The constant A is determined by matching the average slope in the first interval (9000-10,000 BP) to the known value from the calibration curve. This determination of the value of A is equivalent to the determination of the value of $(b-a)$, a procedure discussed in the previous sec- 

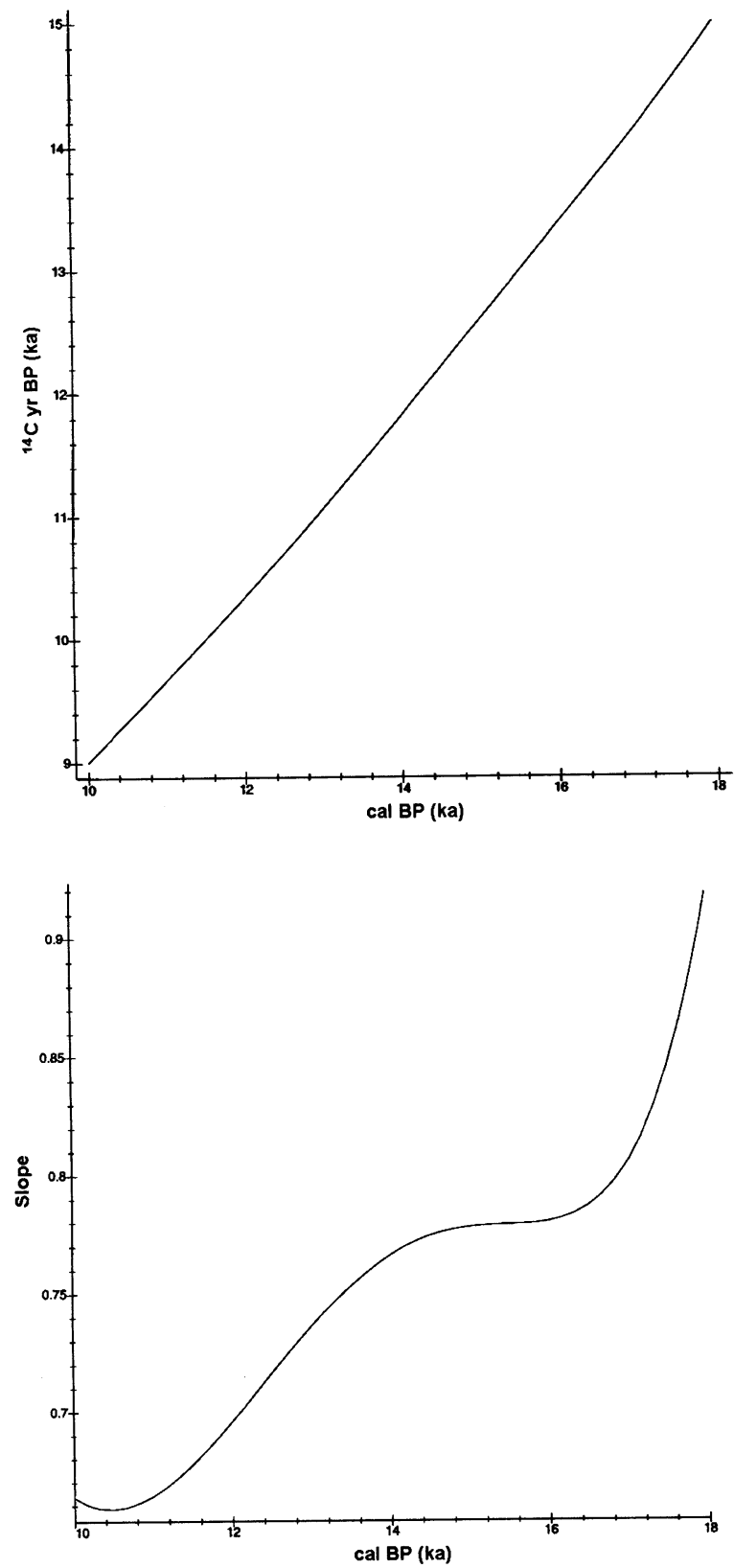

Figure $1{ }^{14} \mathrm{C}$ calibration curve given by Equation (6). The $\mathrm{X}$-axis is the true, calibrated date, while the $\mathrm{Y}$-axis is the uncalibrated ${ }^{14} \mathrm{C}$ date. Both dates are measured in $\mathrm{ka}$.

Figure 2 The first derivative, or slope, of the calibration curve given by Equation (6), plotted as a function of the true age in ka. Note that the slope is dimensionless.

tion. In Table 1 we also give the expected value of the average slope for each interval; this expected value is computed, using the calibration curve, from the $\Delta y$ and the $\Delta x$ of each interval by the simple relation

$$
s_{i}=\frac{\Delta y}{\Delta x} .
$$


Table 1 Results of the numerical experiment with 2400 events. The number of events in each time interval is given; this number is divided by 2400 to give $p_{i}$. The experimentally determined average slope for each interval is then given by $s_{i}$, whereas the expected average slope (computed from the calibration curve) is given in the last column. No value for $s_{i}$ is predicted in the first time interval, since the constant $\mathrm{A}$ was determined by using the $p_{i}$ for this interval. The accuracy of the method can be judged by comparing the numbers in the last 2 columns.

\begin{tabular}{r|c|c|c|c}
\hline Interval & $\begin{array}{c}\text { Number of } \\
\text { events }\end{array}$ & $p_{i}$ & $s_{i}$ & Expected $s_{i}$ \\
\hline $9-10 \mathrm{ka}$ & 430 & .179 & - & .664 \\
$10-11 \mathrm{ka}$ & 432 & .180 & .661 & .705 \\
$11-12 \mathrm{ka}$ & 412 & .172 & .692 & .754 \\
$12-13 \mathrm{ka}$ & 376 & .157 & .758 & .777 \\
$13-14 \mathrm{ka}$ & 370 & .154 & .772 & .783 \\
$14-15 \mathrm{ka}$ & 380 & .158 & .753 & .843 \\
\hline
\end{tabular}

Table 2 Results of the numerical experiment with 4800 events. Notation is the same as in Table 1, except that $p_{i}$ is computed by dividing the number of events by 4800 .

\begin{tabular}{c|c|c|c|c}
\hline Interval & $\begin{array}{c}\text { Number of } \\
\text { events }\end{array}$ & $p_{i}$ & $s_{i}$ & Expected $s_{i}$ \\
\hline $9-10 \mathrm{ka}$ & 901 & .188 & - & .664 \\
$10-11 \mathrm{ka}$ & 860 & .179 & .698 & .705 \\
$11-12 \mathrm{ka}$ & 788 & .164 & .762 & .754 \\
$12-13 \mathrm{ka}$ & 765 & .159 & .786 & .777 \\
$13-14 \mathrm{ka}$ & 782 & .163 & .767 & .783 \\
$14-15 \mathrm{ka}$ & 704 & .147 & .850 & .843 \\
\hline
\end{tabular}

Table 2 shows results for a larger sample of 4800 randomly generated events. The crucial check on the method is a comparison of the last 2 columns in the tables, which contain the experimentally estimated average slope and the actual average slope. We can see that in Table 1 the trend of an increasing slope is roughly captured by the data. The results in Table 2 are noticeably better.

We can now discuss random fluctuations, and how they affect the accuracy of this method. If $M$ is total number of points (in our case 2400 or 4800 ) and $\bar{N}_{i}$ is the average number of points expected in a given interval (in our case 1 of our 6 intervals), then it is easy to show that

$$
\Delta N_{i}=\left(\bar{N}_{i}\right)^{\frac{1}{2}}\left(1-\frac{\bar{N}_{i}}{M}\right)^{\frac{1}{2}} .
$$

Here $\Delta N_{i}$ is the variance in $N_{i}$. Thus, to a good approximation we can say that

$$
\Delta N_{i} \approx\left(\bar{N}_{i}\right)^{\frac{1}{2}} .
$$

Thus, we can say that

$$
\Delta p_{i} \approx \frac{p_{i}}{\left(\bar{N}_{i}\right)^{\frac{1}{2}}}
$$

and 


$$
\Delta s_{i} \approx \frac{(2)^{\frac{1}{2}} s_{i}}{\left(\bar{N}_{i}\right)^{\frac{1}{2}}}
$$

The $\sqrt{ } 2$ factor in the last equation appears when we take into account, in an approximate manner, the additional variance due to the uncertainty in the value of the experimentally determined A. So, for the 2400-event sample, we estimate that the uncertainty in slope determination is roughly $\Delta s_{i} \approx$ 0.049 , while for the 4800 -event sample we estimate $\Delta s_{i} \approx 0.035$. So, for a given number of events in our sample, a certain resolution in slope determination is expected.

\section{CONCLUSION}

We have discussed the general principles behind the Date Distribution method for calibrating the ${ }^{14} \mathrm{C}$ time scale, and worked through a numerical experiment to show how it could work in practice. It is clear that a crucial difficulty in applying the method lies in assembling a sample set of ${ }^{14} \mathrm{C}$ dates with the correct statistical properties. If the method seems promising enough for further effort, we hope archaeological and geological insight can be brought to bear in assembling such a sample.

\section{ACKNOWLEDGMENTS}

I am grateful to David Elmore, Nick Giordano, and Darryl Granger for useful discussions.

\section{REFERENCE}

Stuiver M., Reimer PJ, Bard E, Beck JW, Burr GS, Hughen KA, Kromer B, McCormac G, van der Plicht J, Spurk M. 1998 INTCAL98 radiocarbon age calibration, 24,000-0 cal BP. Radiocarbon 40:1041-77. 